surgical prophylaxis. The most frequent antimicrobials were cefuroxime and cefotaxime. The AMS team found that they would have stopped or switched IV antibiotics to oral at 48 hours in $36 \%$ of cases when they had actually been continued, this was most common for patients receiving IV antibiotics for pneumonia, UTI and surgical prophylaxis. Six out of 78 patients were found to be eligible for OPAT at the time of review.

Conclusions This audit demonstrated that a systematic 48 hours review by a dedicated antimicrobial stewardship team could improve adherence with the Department of Health "Start Smart Then Focus" guidance ${ }^{1}$ and improve patient care by stopping antibiotics early or switching to oral therapy. This study also estimates that combining this systematic review at 48 hours with an improved OPAT service could save 1800 bed days per year locally.

\title{
REFERENCE
}

1 Department of Health. Antimicrobial Stewardship: "Start Smart - Then Focus." Guidance for antimicrobial stewardship in hospitals (England). Gateway reference: 16853. London. HMSO; 2011.

\section{P 34 HOW A CLINICAL ANTIMICROBIAL STEWARDSHIP TEAM COULD IMPROVE ANTIBIOTIC USE AT A CHILDREN'S HOSPITAL}

Hayley Sommerville, ${ }^{1}$ David Sharpe, ${ }^{2}$ Stephane Paulus ${ }^{2} .{ }^{1}$ University of Liverpool; ${ }^{2}$ Alder Hey Children's NHS Foundation Trust

10.1136/archdischild-2015-308634.42

Aim The Department of Health have published guidelines which provide an outline for evidence-based Antimicrobial Stewardship (AMS) in secondary healthcare settings. ${ }^{1}$ The guidance states antimicrobials should not be started without clinical evidence of a bacterial infection and that all patients should be reviewed at 48 hours to make a clear plan of action; the "Antimicrobial Prescribing Decision". The aim of this audit is to establish how often the 48 hour review takes place at our centre, and to evaluate the appropriateness of the decisions made at 48 hours, and estimate the impact of establishing a dedicated AMS team on patient care.

Methods Data collection took place from Jan to May 2013. Ten wards (mix of medical and surgical) were visited on five occasions over that period. Information was collected from patients prescribed IV antibiotics for 48 hours or longer, and included basic demographic data, diagnosis, antibiotic prescribed and dose, and whether a 48 hour review had taken place. The data was reviewed by a virtual AMS team (Infectious Diseases (ID) consultant and ID pharmacist) and each patient underwent a 48 hour "Antimicrobial Prescribing Decision", which was compared to the clinical decision. In addition the team assessed the eligibility for the patient for Outpatient Parenteral Antimicrobial Therapy (OPAT).

Results Of the 533 patients screened, 78 (15\%) had been prescribed antibiotics for more than 48 hours. The most common indications for IV antibiotics were pneumonia, sepsis and 